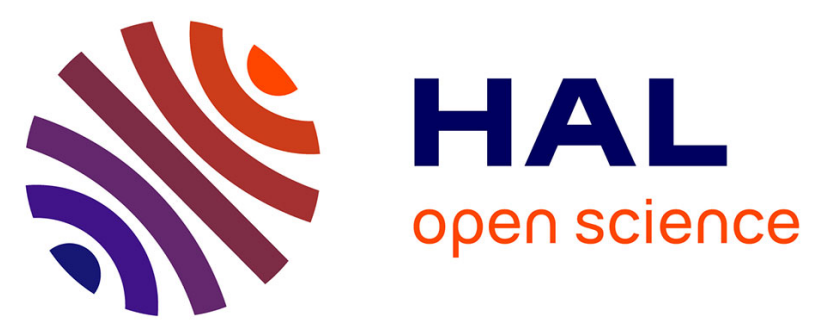

\title{
Gene expression profiling and prediction of response to hormonal neoadjuvant treatment with anastrozole in surgically resectable breast cancer
}

Maurizia Mello-Grand, Vijay Singh, Chiara Ghimenti, Maria Scatolini, Lea Regolo, Enrico Grosso, Alberto Zambelli, Gian Antonio Prada, Laura Villani, Vittorio Fregoni, et al.

\section{To cite this version:}

Maurizia Mello-Grand, Vijay Singh, Chiara Ghimenti, Maria Scatolini, Lea Regolo, et al.. Gene expression profiling and prediction of response to hormonal neoadjuvant treatment with anastrozole in surgically resectable breast cancer. Breast Cancer Research and Treatment, 2010, 121 (2), pp.399411. 10.1007/s10549-010-0887-y . hal-00535452

\section{HAL Id: hal-00535452 https://hal.science/hal-00535452}

Submitted on 11 Nov 2010

HAL is a multi-disciplinary open access archive for the deposit and dissemination of scientific research documents, whether they are published or not. The documents may come from teaching and research institutions in France or abroad, or from public or private research centers.
L'archive ouverte pluridisciplinaire $\mathbf{H A L}$, est destinée au dépôt et à la diffusion de documents scientifiques de niveau recherche, publiés ou non, émanant des établissements d'enseignement et de recherche français ou étrangers, des laboratoires publics ou privés. 


\title{
Gene expression profiling and prediction of response to hormonal neoadjuvant treatment with anastrozole in surgically resectable breast cancer
}

\author{
Maurizia Mello-Grand • Vijay Singh - Chiara Ghimenti - Maria Scatolini • \\ Lea Regolo - Enrico Grosso - Alberto Zambelli • Gian Antonio Da Prada • \\ Laura Villani · Vittorio Fregoni · Paola Baiardi · Silvia Marsoni • \\ William R. Miller • Alberto Costa $\cdot$ Giovanna Chiorino
}

Received: 2 March 2010/Accepted: 1 April 2010/Published online: 29 April 2010

(C) Springer Science+Business Media, LLC. 2010

\begin{abstract}
Aromatase inhibition (AI) is the most effective endocrine treatment for breast cancer in post-menopausal patients, but a percentage of hormone receptor-positive cancers do not benefit from such therapy: for example, about $20 \%$ of patients treated with anastrozole do not respond and it is still impossible to accurately predict sensitivity. Our main goal was to identify a robust expression signature predictive of response to neoadjuvant treatment with anastrozole in patients with $\mathrm{ER}+$ breast cancer. At the same time, we addressed the question of delineating treatment effects and possible mechanisms of intrinsic resistance occurring in non-responder patients. We analyzed the transcriptome of 17 tru-cut biopsies before treatment and 13 matched surgical samples after 3 months
\end{abstract}

Maurizia Mello-Grand and Vijay Singh contributed equally to the work.

Electronic supplementary material The online version of this article (doi:10.1007/s10549-010-0887-y) contains supplementary material, which is available to authorized users.

M. Mello-Grand · V. Singh · C. Ghimenti · M. Scatolini ·

E. Grosso - G. Chiorino $(\square)$

Cancer Genomics Laboratory, Fondazione "Edo ed Elvo

Tempia Valenta", Via Malta 3, 13900 Biella, Italy

e-mail: giovanna.chiorino@fondoedotempia.it

L. Regolo $\cdot$ A. Costa

Unit of Senology, IRCCS Fondazione

"Salvatore Maugeri", Pavia, Italy

A. Zambelli · G. A. Da Prada - V. Fregoni

Unit of Medical Oncology, IRCCS Fondazione

"Salvatore Maugeri", Pavia, Italy

L. Villani

Unit of Pathology, IRCCS Fondazione

"Salvatore Maugeri", Pavia, Italy treatment with anastrozole taken from ER+ breast tumors. Molecular profiles were related to clinical response data. Treatment with anastrozole was associated with a decreased expression of genes relating to cell proliferation and an increased expression of genes relating to inflammatory processes. There was also an enrichment of induction of T-cell anergy, positive regulation of androgen signalling, synaptic transmission and vesicle trafficking in non-responders, and of cell cycle inhibition and induction of immune response in responders. We identified an expression signature of 77 probes ( 54 genes) that predicted response in $100 \%$ of our cases. Five of them were able to accurately predict response on an independent dataset $(P=0.0056)$ of $52 \mathrm{ER}+$ breast cancers treated with letrozole. Ten fixed independent samples from the anastrozole study were also used for RT-qPCR validations. This study suggests that a relative small number of genes analysed in a pre-treatment biopsy may identify patients likely to respond to AI neoadjuvant treatment. This may have practical utility translatable to the clinics. Furthermore, it

\footnotetext{
P. Baiardi

Consorzio per le valutazioni Biologiche e Farmacologiche (CVBF), Università degli Studi di Pavia e IRCCS Fondazione

"Salvatore Maugeri", Pavia, Italy

S. Marsoni

Southern Europe New Drug Organization, Milano, Italy

W. R. Miller

Breast Research Group, University of Edinburgh,

Edinburgh, UK

A. Costa

Ente Ospedaliero Cantonale, Lugano, Switzerland
} 
delineates novel mechanisms of intrinsic resistance to $\mathrm{AI}$ therapy that could be further investigated in order to explore circumventing treatments.

Keywords Breast cancer - Aromatase inhibitors · Gene expression profiling · Neoadjuvant treatment . Response prediction - Intrinsic resistance

\section{Introduction}

Breast cancer represents the most widespread tumor in Western women: incidence in Italy is more than 30,000 new cases per year. Nearly $80 \%$ of human mammary carcinomas express estrogen receptor (ER) at clinically significant levels [1]. Estrogen-bound ER induces the expression of peptide growth factors that are responsible for cancer cell proliferation.

In estrogen-dependent breast tumors, control of tumor growth can be achieved by treatment with estrogen antagonists that block the binding of the hormone to ER: tamoxifen has been the predominant treatment for the past two decades $[1,2]$. In postmenopausal women, aromatase inhibitors (AIs) have shown to be more effective than tamoxifen as adjuvant therapy [3-6]. Aromatase is the enzyme that synthesizes estrogens by converting C19 androgens to aromatic C18 estrogenic steroids. Experimental results indicate that autocrine produced estrogen plays a more important role than circulating extradiol in breast tumor promotion: aromatase is expressed in breast tumor tissue (both cancer and surrounding adipose stromal cells) probably at a higher level than in normal breast tissue [7, 8]. In hormone dependent breast cancer, neoadjuvant therapy with third generation AI therapy (anastrozole, letrozole, exemestane) reduces proliferation, causes cell death and produces tumor regression $[9,10]$. Although of proven clinical efficacy, such therapies are still associated with non-response in about $20 \%$ of cases [11]. Up to now, it is still impossible to accurately predict who will respond to such treatments.

Furthermore, little is known of (i) the effects of antiaromatase treatment in vivo, (ii) how these effects vary between tumors and (iii) whether the molecular changes fully reflect and are responsible for the clinical response. Preoperative treatment followed by surgical resection provides an excellent opportunity to correlate baseline molecular markers measured in a diagnostic needle biopsy with subsequent response to treatment, and to delineate molecular changes induced by the treatment itself.

A pivotal study on anastrozole treatment in 12 patients identified 298 genes differentially expressed between partial response and progressive disease [12]. Signatures correlated to response to neoadjuvant treatment have been also identified for exemestane, although on a very small patient cohort, and letrozole [13, 14].
Our goals were to (i) examine changes in gene expression with anastrozole therapy and (ii) identify genetic signatures that might distinguish between ER+ post-menopausal breast cancers benefiting from treatment and those not. To elucidate mode of action, we compared the transcriptional profile before and after three months of anastrozole treatment in 13 patients. In order to derive a predictive signature, we analysed global gene expression profiles of $17 \mathrm{ER}+$ breast cancers before neoadjuvant therapy and associated them to response values. Genes predictive of response were then tested on a publicly available independent dataset [14] and on an independent cohort of fixed tissues.

\section{Methods}

Patients

All tumor samples and clinical data were collected with the Fondazione S. Maugeri ethical committee's approval and patients' informed consent. The 17 patients, enrolled into this study between July 2004 to November 2007, were post-menopausal and had breast cancer stage T2 or T3, greater than 2.5 centimeters, any lymph node status and no distant metastasis. All the tumors were HER2/neu- and $\mathrm{ER}+/ \mathrm{PgR}+$, except 2 that were $\mathrm{ER}+/ \mathrm{PgR}-$ (Table 1). They received neoadjuvant therapy with Anastrozole (Arimidex $^{\mathrm{TM}}$, Astra Zeneca) $1 \mathrm{mg}$ po/die for three months. Clinical response was evaluated by serial tumor clinical examination and mammary ultrasound bidimensional measurements, performed by single operator (LR) before, during and after treatment. Patients with decrease in tumor volume greater than or equal to $30 \%$, according to RECIST criteria [15], were classified as responders.

\section{Microarray hybridization and data processing}

Samples were removed by tru-cut biopsy or surgical resection and stored in RNAlater (Ambion Inc., Austin, TX) for processing. Tissues were homogenized and total RNA was isolated using TriReagent (Sigma-Aldrich, St. Louis, MO) and RNeasy Mini Kit (Qiagen, Dusseldorf, Germany). mRNA amplification and labeling, quality control and microarray hybridization were carried out as previously described [16]. Two oligonucleotide glass arrays (Human Whole Genome Oligo Microarray 1x44K or 4x44K, Agilent Technologies, Palo Alto, CA) were utilized for each sample, using the Human Universal Reference Total RNA (Clontech, Palo Alto, CA) as baseline and dyeswap duplication.

Slides were scanned with the Agilent G2505B scanner and the TIFF images loaded into the Feature Extraction 
Table 1 Pathophysiological characteristics and percentage of response to therapy of analyzed patients

\begin{tabular}{|c|c|c|c|c|c|c|c|c|c|c|c|c|c|c|c|c|c|}
\hline \multirow[t]{2}{*}{ Patient } & \multirow{2}{*}{$\begin{array}{l}\text { Age } \\
\text { (years) }\end{array}$} & \multirow{2}{*}{$\begin{array}{l}\text { Initial size } \\
(\mathrm{mm})\end{array}$} & \multicolumn{3}{|c|}{ Tru-cut } & \multicolumn{6}{|c|}{ Surgical sample } & \multicolumn{3}{|c|}{ Stage } & \multicolumn{2}{|c|}{ Lymph-node } & \multirow{2}{*}{$\begin{array}{l}\% \\
\text { Response }\end{array}$} \\
\hline & & & $\begin{array}{l}\text { ER } \\
(\%)\end{array}$ & $\begin{array}{l}\mathrm{PgR} \\
(\%)\end{array}$ & $\begin{array}{l}\text { ki67 } \\
(\%)\end{array}$ & $\begin{array}{l}\text { Size } \\
(\mathrm{mm})\end{array}$ & Histotype & Grading & $\begin{array}{l}\text { ER } \\
(\%)\end{array}$ & $\begin{array}{l}\mathrm{PgR} \\
(\%)\end{array}$ & $\begin{array}{l}\text { ki67 } \\
(\%)\end{array}$ & $\mathrm{T}$ & $\mathrm{N}$ & $\mathrm{M}$ & $\begin{array}{l}\text { Positive } \\
\mathrm{N}^{\circ}\end{array}$ & $\begin{array}{l}\text { Total } \\
\mathrm{N}^{\circ}\end{array}$ & \\
\hline $10051 *$ & 70 & 29 & 80 & 0 & 10 & 25 & ILC & 2 & 80 & 5 & 5 & 2 & 1 & 0 & 1 & 20 & 14 \\
\hline $10054 *$ & 52 & 37 & 80 & 80 & 8 & 30 & ILC & 2 & 60 & 40 & 40 & $1 \mathrm{C}$ & 1 & 0 & 1 & 14 & 19 \\
\hline $10052 *$ & 80 & 25 & 80 & 80 & 8 & 20 & IDC & 2 & 80 & 60 & 5 & $1 \mathrm{C}$ & 1 & 0 & 1 & 7 & 20 \\
\hline $10018 *$ & 74 & 30 & 80 & 60 & 8 & 23 & ILC & 2 & 80 & 20 & 10 & 2 & 1 & 0 & 2 & 15 & 23 \\
\hline $10000^{*}$ & 70 & 53 & 70 & 40 & 10 & 40 & IDC & 3 & 70 & 20 & 8 & 3 & 1 & 0 & 14 & 14 & 24 \\
\hline $10028 *$ & 73 & 26 & 80 & 70 & 5 & 19 & IDC & 1 & 80 & 60 & 5 & 2 & 1 & 0 & 2 & 10 & 27 \\
\hline $10056^{*}$ & 75 & 39 & 70 & 10 & 15 & 28 & IDC & 1 & 80 & 10 & 8 & 2 & 0 & 0 & 0 & 22 & 28 \\
\hline $10064 *$ & 83 & 25 & 80 & 80 & 5 & 18 & IDC & 1 & 80 & 40 & 5 & $1 \mathrm{C}$ & $\mathrm{NV}$ & 1 & $\mathrm{NV}$ & NV & 28 \\
\hline 10040 & 72 & 28 & 80 & 80 & 5 & 19 & ILC & 2 & 80 & 70 & 5 & $1 \mathrm{C}$ & NV & $\mathbf{0}$ & NV & NV & 32 \\
\hline $10026^{*}$ & 76 & 30 & 80 & 80 & 10 & 20 & IDC & 2 & 90 & 70 & 10 & 2 & NV & $\mathbf{0}$ & NV & NV & 33 \\
\hline $10010 *$ & 66 & 25 & 70 & 90 & 8 & 16 & ILC & 2 & 80 & 80 & 8 & $1 \mathrm{C}$ & 0 & $\mathbf{0}$ & $\mathbf{0}$ & 8 & 36 \\
\hline 10036 & 66 & 25 & 60 & $\mathbf{0}$ & 5 & 16 & ILC & 2 & 80 & $\mathbf{0}$ & 10 & 2 & 0 & $\mathbf{0}$ & $\mathbf{0}$ & 8 & 36 \\
\hline $10017^{*}$ & 73 & 26 & 90 & 90 & 40 & 16 & IDC & 2 & 90 & 80 & 10 & $1 \mathrm{C}$ & $\mathbf{0}$ & $\mathbf{0}$ & $\mathbf{0}$ & 12 & 38 \\
\hline 10039 & 85 & 27 & 80 & 80 & 10 & 16 & ILC & 3 & 80 & 70 & 5 & $1 \mathrm{C}$ & NV & $\mathbf{0}$ & NV & NV & 40 \\
\hline 10037 & 65 & 25 & 80 & 80 & 5 & 14 & IDC & 2 & 80 & 70 & 5 & $1 \mathrm{C}$ & 0 & $\mathbf{0}$ & $\mathbf{0}$ & 6 & 44 \\
\hline 9999* & 78 & 40 & 80 & 70 & 5 & 21 & IDC & 2 & 80 & 40 & 5 & 2 & 1 & $\mathbf{0}$ & 8 & 9 & 47 \\
\hline $10061 *$ & 79 & 27 & 90 & 90 & 10 & 13 & ILC & 2 & 90 & 80 & 10 & $1 \mathrm{C}$ & NV & $\mathbf{0}$ & NV & NV & 52 \\
\hline
\end{tabular}

The * indicates patients with both pre- and post-treatment samples available for hybridization

ILC infiltrating lobular carcinoma, IDC infiltrating ductal carcinoma, $N V$ not valuable

software (Agilent Technologies). Raw data were processed using the statistical computing software " $\mathrm{R}$ " and packages from Bioconductor (www.bioconductor.org); "limma" was used for preprocessing and differential expression analysis. Raw intensities were first background corrected ("normexp" method, with an offset value of 50 added to red and green intensities so that log-ratios are shrunk towards zero at lower intensities). In order to set log-ratios average to zero within each array and to have similar log-ratio distributions across all arrays, "loess" and "Aquantile" normalization were performed [17].

Gene ontology analysis was accomplished with GOEAST (http://omicslab.genetics.ac.cn/GOEAST/) and pathway analysis with "MetaCore 5.0" (GeneGO, St. Joseph, MI).

Signature extraction and evaluation

Genes differentially expressed by the treatment were obtained as previously described [18]. The difference matrix was defined as the difference between post and pre treatment expression matrices. To derive a predictive signature, we first calculated the Pearson correlation coefficient between tru-cut expression and response data, used as continuous variable. Then the two sample modified $t$-test available in the "limma" package was performed, using response data as a binary variable. The best predictors from the two lists were selected using the rank product method.
For sample classification, leave-one-out cross validation procedure was applied. Briefly, for each list of predictors, the following procedure was carried out: one sample was leaved out and two centroids, one representing responders and the other non-responders, were derived by kmeans clustering and response status was assigned based on majority rule. Then the sample initially leaved out was assigned a class based on Euclidean distance from the two centroids. The procedure was repeated 17 times leaving out each time one sample from centroid calculation and the number of correct prediction reflects the performance of the signature.

To validate the procedure, radical leave-one-out cross validation was performed, where the whole process of signature derivation was repeated leaving each time one sample out of the analysis and the derived signature was used to classify the sample initially leaved out.

The standalone JAVA-based Signature Evaluation Tool (http://microarray.ym.edu.tw) was used to predict responsiveness on test samples in radical leave-one-out cross validation and on the independent dataset (GSE5462).

\section{RT-qPCR}

Total RNA was extracted from an independent cohort of 10 formalin-fixed, paraffin-embedded breast cancer pre-treatment biopsies using Recover All Nucleic Acid Isolation kit 
(Ambion Inc., Austin, TX), according to manufacturer instructions.

Reverse Transcriptase quantitative Polymerase Chain Reaction (RT-qPCR) was performed as previously described [19] to validate a subset of differently expressed transcripts (CXCL3, GSS, FBXL18 and DUSP1) identified by microarray analysis on fresh samples. Primers sequences are available in Supplementary Table 1. First strand cDNA synthesis was performed using $200 \mathrm{ng}$ of total RNA. The relative abundance of transcripts was established using the mean expression of TBP and RPLP0 for normalization of input cDNA.

\section{Results}

A flow chart outlying the statistical methods used for analyzing expression data is shown in Fig. 1.

Change in gene expression with therapy

The molecular profiles of all the 17 patients listed in Table 1 were analyzed before therapy, and for 13 of them (identified by a star after the patient's code) we could also evaluate the change in gene expression with treatment. Three different approaches were used to find probes differentially expressed between pre and post-treatment samples [18]: frequency of change, which measures the consistency by which a probe is up or down-regulated; magnitude of change, which identifies probes having greatest median fold change across all patients; statistically significant change, which identifies probes most significantly changed with therapy, applying two sample paired Student $t$-test.

Combining the lists from the three methods resulted in 634 down-regulated and 646 up-regulated probes, 78 (47 up and 31 down-regulated) of which were reported differentially expressed by all the three methods (Table 2) Venn diagrams representing overlaps between the three lists are shown in Fig. 2. Hierarchical clustering on 1280 probes using the difference matrix was not able to separate responders from non-responders in a statistically significant manner (Supplementary Figure 1). Differentially expressed probes were compared with a list of 2897 estrogen-regulated genes [20], found modulated in response to estrogens and also present on Agilent 44K array. Hypergeometric test showed that our list is significantly enriched in estrogenregulated genes (Fisher exact test $P$ value $=2.2 \times 10^{-16}$ ), as revealed by Metacore analysis, too (data not shown).

In order to understand the functional characteristics of genes changed with treatment, gene ontology analysis was performed separately for down and up-regulated probes. The down-regulated list is basically enriched in terms related to cell cycle (Supplementary Tables 8 and 9), as cell cycle phase, M-phase, mitotic cell cycle, cell cycle, mitosis cell division and regulation of mitotic cell cycle. Other overrepresented GO terms were response to DNA damage stimulus, DNA replication and DNA integrity checkpoint. GO analysis of up-regulated genes showed that this list is more heterogeneous and less specific in terms of functions.

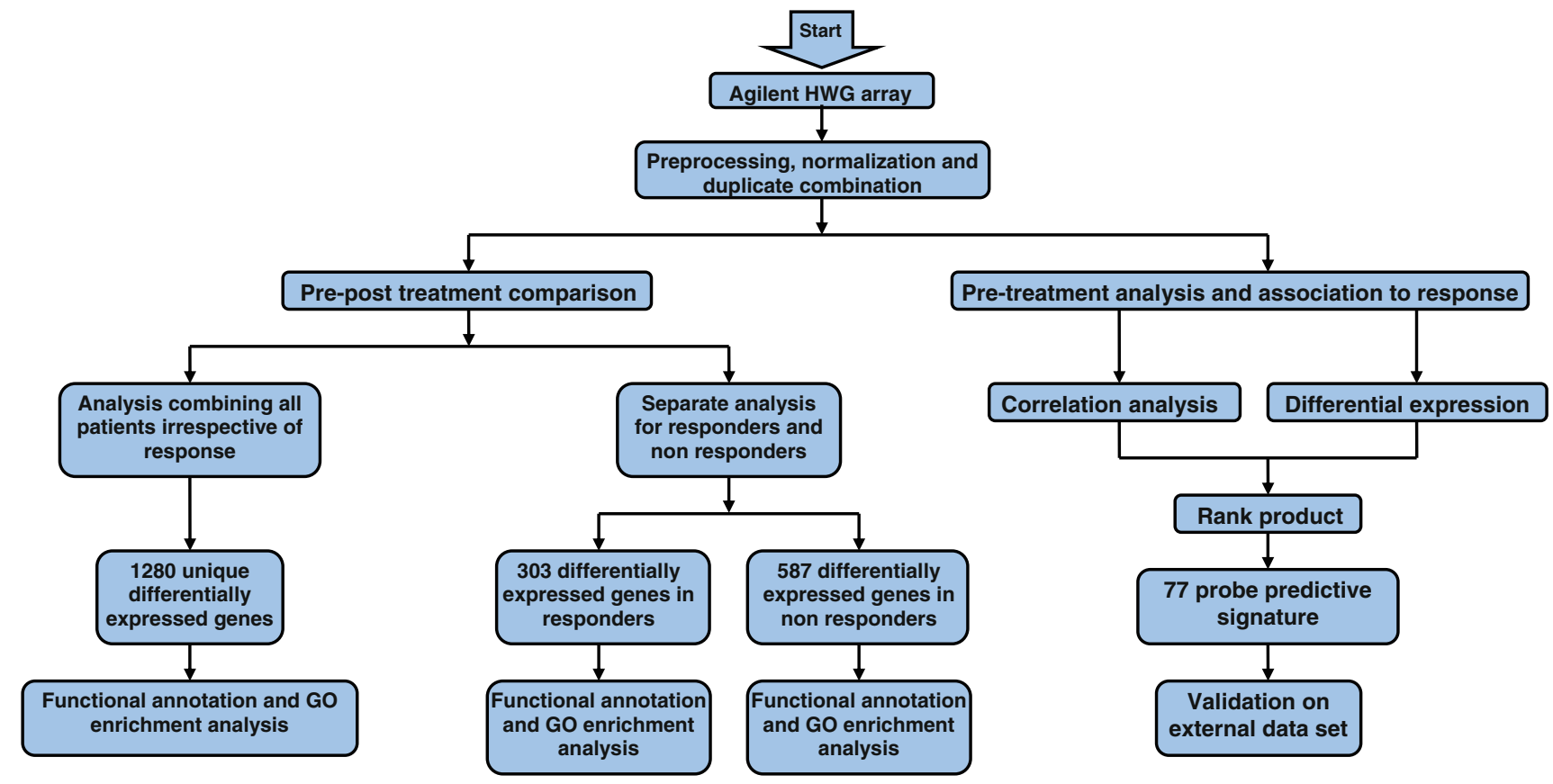

Fig. 1 Flow-chart of data processing and analysis 
Table 278 probes changed with treatment and found by all three statistical methods

\begin{tabular}{|c|c|}
\hline & Down-regulated gene description \\
\hline \multicolumn{2}{|l|}{ Gene symbol } \\
\hline RAMP & RA-regulated nuclear matrix-associated protein (RAMP) \\
\hline CDCA5 & Cell division cycle associated 5 (CDCA5) \\
\hline TK1 & Thymidine kinase 1 , soluble (TK1) \\
\hline HSPC150 & HSPC150 protein similar to ubiquitin-conjugating enzyme (HSPC150) \\
\hline KIAA0101 & KIAA0101 \\
\hline PGR & Progesterone receptor (PGR) \\
\hline HCAP-G & Chromosome condensation protein G (HCAP-G) \\
\hline FKSG14 & Leucine zipper protein FKSG14 (FKSG14) \\
\hline THC2217339 & Unknown \\
\hline UHRF1 & Ubiquitin-like, containing PHD and RING finger domains, 1 (UHRF1) \\
\hline ВC082981 & cDNA clone IMAGE: 6575075 \\
\hline GREB1 & GREB1 protein (GREB1), transcript variant a \\
\hline MGC24665 & Hypothetical protein MGC24665 (MGC24665) \\
\hline CDT1 & DNA replication factor (CDT1) \\
\hline CENPF & Centromere protein F, 350/400 ka (mitosin) (CENPF) \\
\hline SUSD3 & Sushi domain containing 3 (SUSD3) \\
\hline FLJ12650 & Hypothetical protein FLJ12650 (FLJ12650) \\
\hline ASPM & Asp (abnormal spindle)-like, microcephaly associated (Drosophila) (ASPM) \\
\hline ZWINT & ZW10 interactor (ZWINT), transcript variant 2 \\
\hline RECQL4 & RecQ protein-like 4 (RECQL4) \\
\hline BM039 & Uncharacterized bone marrow protein BM039 (BM039) \\
\hline UBE2C & Ubiquitin-conjugating enzyme E2C (UBE2C), transcript variant 6 \\
\hline C22orf18 & Chromosome 22 open-reading frame 18 (C22orf18), transcript variant \\
\hline RPL23A & UI-H-DH0-aul-h-03-0-UI.s1 NCI_CGAP_DH0 cDNA clone IMAGE:5871026 3', sequence \\
\hline RAN & UI-H-DH0-aum-e-05-0-UI.s1 NCI_CGAP_DH0 cDNA clone IMAGE:5871340 3', sequence \\
\hline THC2162693 & RS24_HUMAN (P16632) 40S ribosomal protein S24 (S19), partial (77\%) \\
\hline RIMS4 & Regulating synaptic membrane exocytosis 4 (RIMS4) \\
\hline AL137566 & cDNA DKFZp586G0321 (from clone DKFZp586G0321) \\
\hline A_32_P54260 & Unknown \\
\hline FLJ10719 & Hypothetical protein FLJ10719 (FLJ10719) \\
\hline \multirow[t]{2}{*}{ THC2089973 } & BU589712 AGENCOURT_8908071 NIH_MGC_141 cDNA clone IMAGE:6388605 5', sequence \\
\hline & Up-regulated gene description \\
\hline FOS & v-fos FBJ murine osteosarcoma viral oncogene homolog (FOS) \\
\hline DUSP1 & Dual specificity phosphatase 1 (DUSP1) \\
\hline TGFB1I4 & Transforming growth factor beta 1 induced transcript 4 (TGFB1I4), transcript variant 1 , \\
\hline MLL3 & cDNA DKFZp686C08112 (from clone DKFZp686C08112) \\
\hline CTGF & Connective tissue growth factor (CTGF) \\
\hline PER3 & Period homolog 3 (Drosophila) (PER3) \\
\hline RAB6A & RAB6A, member RAS oncogene family (RAB6A), transcript variant 1 \\
\hline PLDN & Pallidin homolog (mouse) (PLDN) \\
\hline PLDN & Pallidin homolog (mouse) (PLDN) \\
\hline SNAP23 & Synaptosomal-associated protein, $23 \mathrm{kDa}$ (SNAP23), transcript variant 1 \\
\hline EGR1 & Early growth response 1 (EGR1) \\
\hline A_23_P300563 & Unknown \\
\hline A_23_P31563 & Unknown \\
\hline AL833005 & cDNA DKFZp666D074 (from clone DKFZp666D074) \\
\hline
\end{tabular}


Table 2 continued

\begin{tabular}{|c|c|}
\hline & Up-regulated gene description \\
\hline PRKAR1A & $\begin{array}{l}\text { Protein kinase, cAMP-dependent, regulatory, type I, alpha (tissue specific extinguisher 1) (PRKAR1A), } \\
\text { transcript variant } 1\end{array}$ \\
\hline CYR61 & Cysteine-rich, angiogenic inducer, 61 (CYR61) \\
\hline MLL3 & Myeloid/lymphoid or mixed-lineage leukemia 3 (MLL3) \\
\hline CRIM1 & Cysteine-rich motor neuron 1 (CRIM1) \\
\hline A_24_P101072 & Unknown \\
\hline $\mathrm{SCP} 2$ & Sterol carrier protein $2(\mathrm{SCP} 2)$, transcript variant 1 \\
\hline RHOA & Ras homolog gene family, member A (RHOA) \\
\hline AF130080 & Clone FLC0675] PRO2870 \\
\hline AF130080 & Clone FLC0675 PRO2870 \\
\hline RAB7 & RAB7, member RAS oncogene family (RAB7) \\
\hline ATP6V1C1 & ATPase, $\mathrm{H}+$ transporting, lysosomal $42 \mathrm{kDa}, \mathrm{V} 1$ subunit $\mathrm{C}$, isoform 1 (ATP6V1C1), transcript variant 1 \\
\hline THC2166450 & Unknown \\
\hline PRKAR1A & $\begin{array}{l}\text { Protein kinase, cAMP-dependent, regulatory, type I, alpha (tissue specific extinguisher 1) (PRKAR1A), } \\
\text { transcript variant } 1\end{array}$ \\
\hline CYR61 & Cysteine-rich, angiogenic inducer, 61 (CYR61) \\
\hline KIAA0738 & KIAA0738 gene product (KIAA0738), \\
\hline XTP7 & Protein 7 transactivated by hepatitis B virus $\mathrm{X}$ antigen (HBxAg) (XTP7) \\
\hline MALAT1 & cDNA DKFZp586G1023 (from clone DKFZp586G1023) \\
\hline AK097068 & cDNA FLJ39749 fis, clone SMINT2017599 \\
\hline AA452590 & AA452590 zx39f03.s1 Soares_total_fetus_Nb2HF8_9w cDNA clone IMAGE:788861 3', \\
\hline SON & SON DNA binding protein (SON), transcript variant \\
\hline PUM2 & Pumilio homolog 2 (Drosophila) (PUM2) \\
\hline RABGEF1 & RAB guanine nucleotide exchange factor (GEF) 1 (RABGEF1) \\
\hline PRG1 & Proteoglycan 1, secretory granule, (cDNA clone IMAGE:4688573) \\
\hline CXorf39 & cDNA: FLJ21191 fis, clone COL00104 \\
\hline $\mathrm{EIF} 2 \mathrm{C} 4$ & For KIAA1567 protein \\
\hline SMG1 & PI-3-kinase-related kinase SMG-1 (SMG1), transcript variant 1 \\
\hline NUDT4 & nudix (nucleoside diphosphate linked moiety X)-type motif 4 (NUDT4), transcript variant 2 \\
\hline SMG1 & PI-3-kinase-related kinase SMG-1 (SMG1), transcript variant 1 \\
\hline SMG1 & PI-3-kinase-related kinase SMG-1 (SMG1), transcript variant 1 \\
\hline LOC286170 & cDNA FLJ31058 fis, clone HSYRA2000828 \\
\hline NOTCH2NL & cDNA FLJ11946 fis, clone HEMBB1000709 \\
\hline LOC200030 & Hypothetical protein LOC200030 (LOC200030) \\
\hline AK027225 & cDNA: FLJ23572 fis, clone LNG12403 \\
\hline
\end{tabular}

The up-regulated genes feature terms like MHC protein complex, antigen processing and presentation, phosphoinositide phosphorylation, immune system process and insulin like growth factor binding. The top GO processes, pathway maps and network processes found for the up and down-regulated probes with their $P$-values are listed in Supplementary Table 10 and graphically shown in Fig. 3.

Signature predictive of response

Using the response criteria as described in methods, 9 patients were classified as responders and 8 as non-responders (Table 1). No patient showed disease progression during the treatment. In this limited series of 17 breast cancers it has not been possible to identify any statistically significant association between the response and the base-line expression of ER, PGR, Ki67, or lymph nodes involvement. We only observed that tumors with low PgR and N1 responded less than tumors with high PgR tumors and N0, respectively.

So we applied correlation analysis using pre-treatment expression data and the percentage of response as a continuous variable. The log ratios for each probe across 17 samples and the response data were scaled to have mean zero and standard deviation one. A filter was applied to remove all the probes with more than three missing values or standard deviation less than 0.2 across 17 pre-treatment 


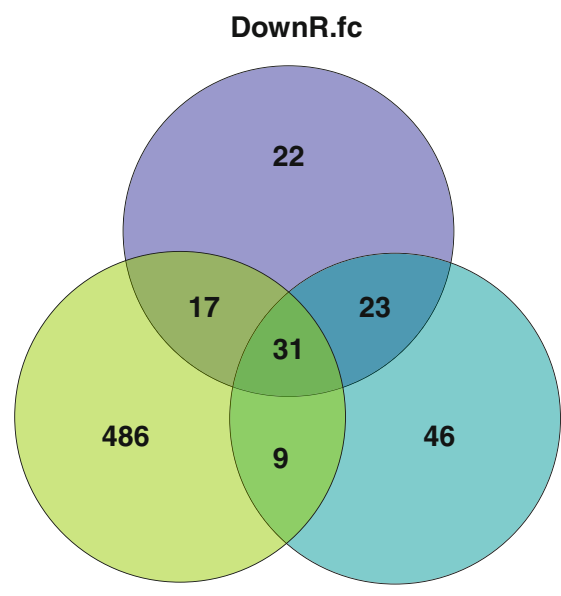

DownR.sc
DownR.mc

Fig. 2 Overlap among differentially expressed probes. Venn diagram representing overlap among down-regulated probes (a) and upregulated probes (b) from three methods. Using the frequency of change approach (fc), 93 down-regulated and 203 up-regulated probes in 10 out of 13 patients were found (Supplementary Tables 2 and 3). Using the magnitude of change $(\mathrm{mc})$ method (change in at least 10
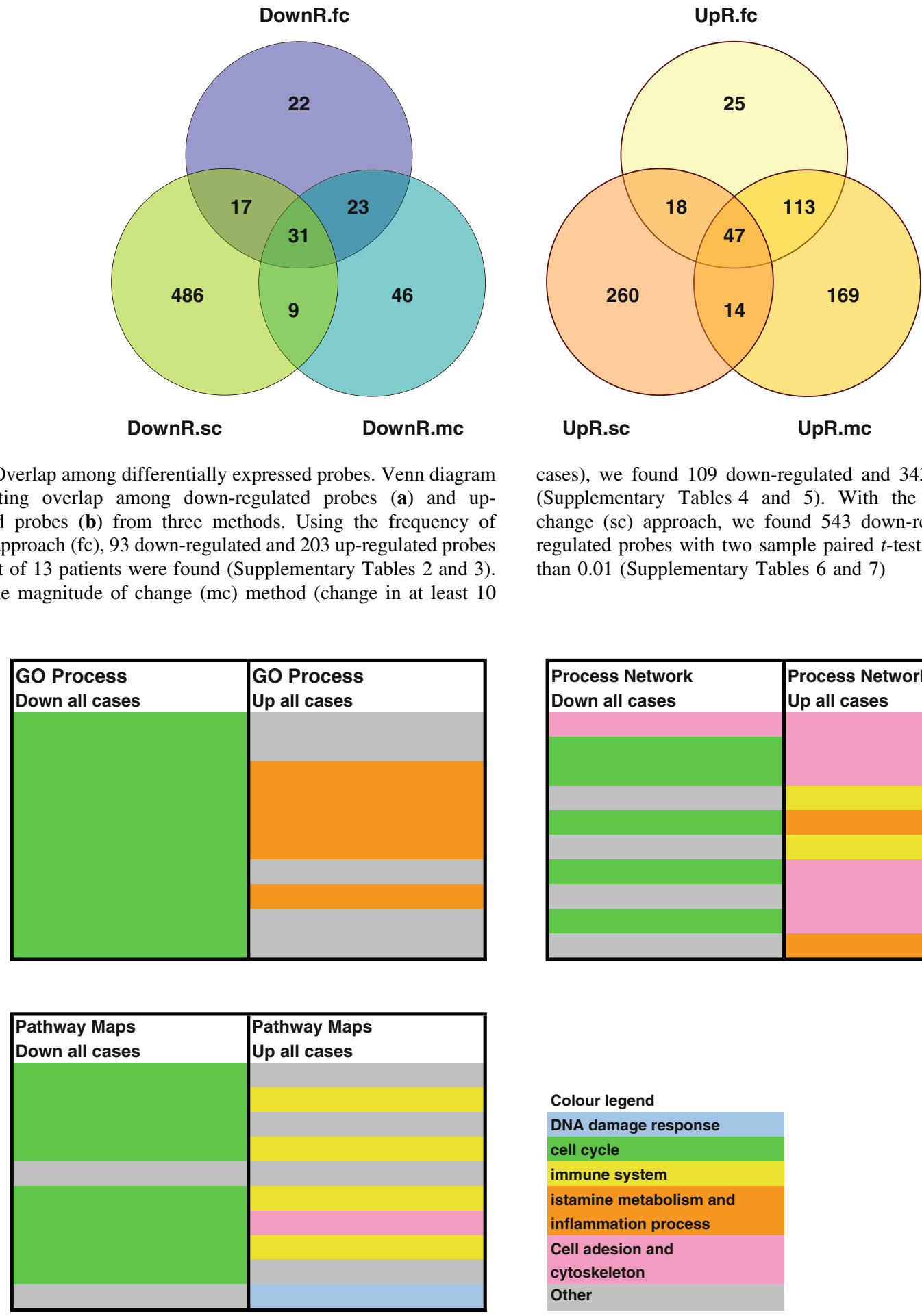

cases), we found 109 down-regulated and 343 up-regulated probes (Supplementary Tables 4 and 5). With the statistical significant change (sc) approach, we found 543 down-regulated and 339 upregulated probes with two sample paired $t$-test adjusted $P$-value less than 0.01 (Supplementary Tables 6 and 7)

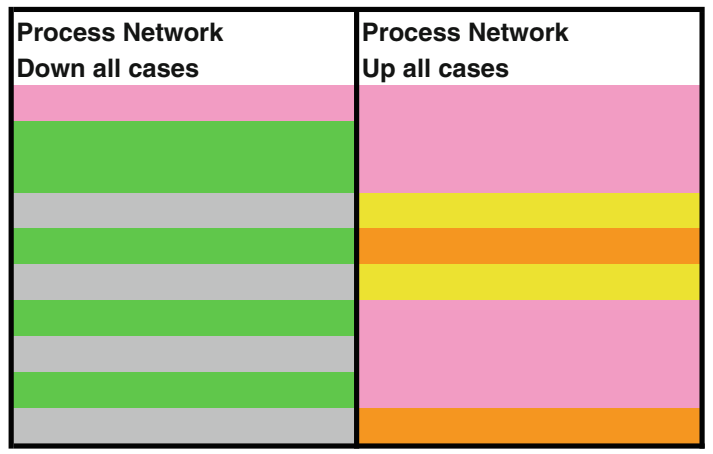

Colour legend

DNA damage response
cell cycle
immune system
istamine metabolism and
inflammation process
Cell adesion and
cytoskeleton
Other

Fig. 3 Go analysis of genes differentially expressed in pre-post treatment samples. Each rectangular represents a single category and is ordered according to ascending $P$-value (as in Supplementary Table 10). Similar pathways or processes have the same color code

samples. After the filtering step, 35,181 probes were selected for further analysis. 159 probes had correlation test $P$-value less than 0.01 . Out of these, 107 were positively correlated and 52 negatively correlated with response. Probes with their correlation and associated $P$-value are listed in Supplementary Table 11. Hierarchical clustering with Euclidean distance over 159 probes classifies patients into 2 distinct groups (Fig. 4): the cluster on the left contains only non-responders, while the one on the right contains all the responders and two patients with response 
Fig. 4 Hierarchical clustering with Euclidean as distance measure over the 159 probes most correlated with response classifies patients into 2 distinct groups. Blue rectangle $=$ non-responders; red rectangle $=$ responders

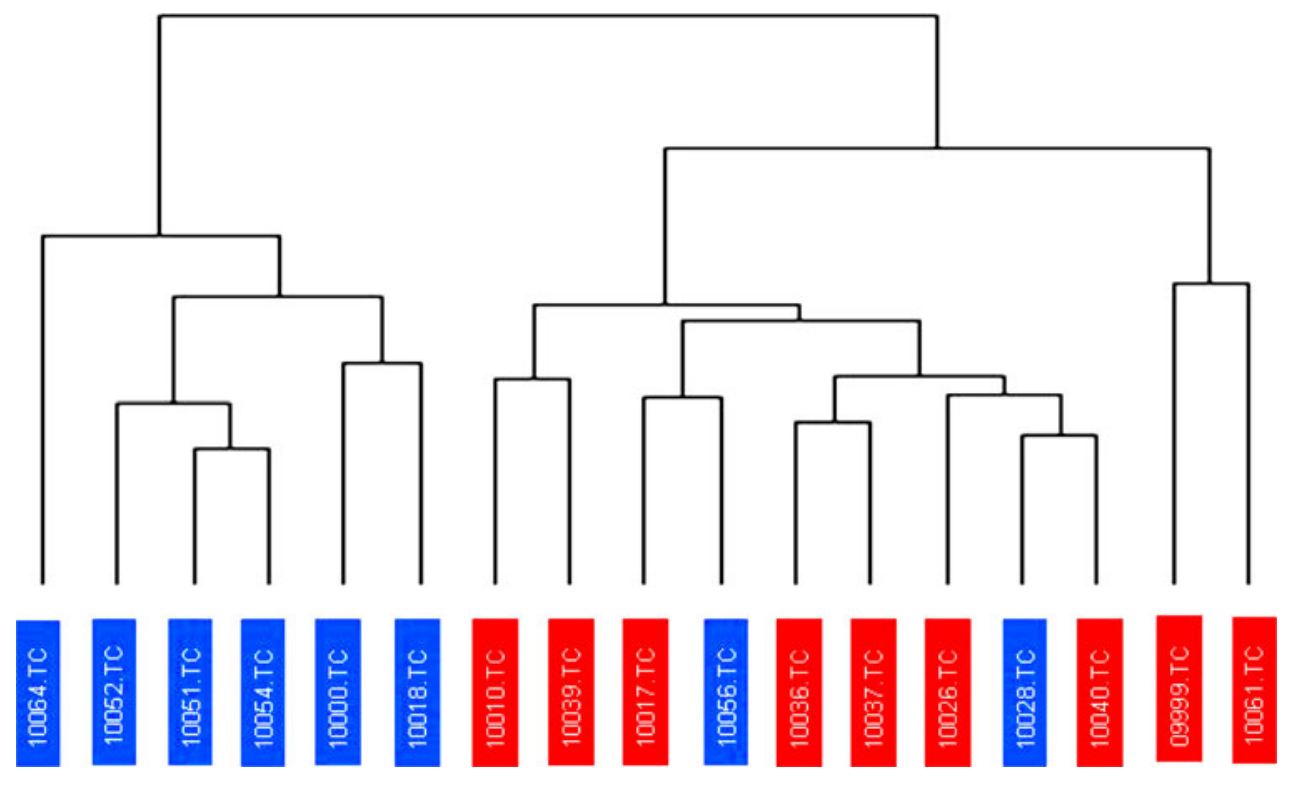

values slightly below the cut-off. Repeated runs of $k$-means clustering find similar grouping when patients are classified in two clusters. Leave-one-out cross validation (with 2-means clustering) correctly predicts responsiveness of 14 patients out of 17 (Fisher $P$-value $=0.015$ ).

Then two Sample $t$-test was performed on pre-treatment samples to identify genes differentially expressed between responders and non-responders. Analysis selected 234 probes with $P$-value $<0.01$ (Supplementary Table 12). Hierarchical clustering over these probes separated responders from non-responders with one exception (data not shown). The patients misclassified had response percentage 27 and was actually placed into the responsive cluster group. $k$-Means clustering produced the same results when samples are classified into two groups. Leaveone-out cross validation correctly predicts 16 patients (Fisher $P$-value $=0.0004)$. The wrongly predicted sample was the same previously misclassified case.

The predictive signatures identified by the two methods have 39 probes in common. In order to have best compromise for informative probes, the two signature lists were combined using the rank product method. Probes were ordered according to the product of their ranks. The top 77 probes were selected as final signature (54 unique genes, Tables 3 and 4), as they performed with $100 \%$ accuracy in separating responders from non-responders in leave-oneout cross validation. To validate the procedure, radical leave-one-out cross validation was performed and SET (Signature Evaluation Tool) was used to predict the responsiveness of the test sample. We were able to correctly predict responsiveness for $82 \%$ of the samples (Fisher exact $P$-value $=0.015$ )

The 77-probe signature predictive of response to anastrozole was then tested on a totally independent dataset
(GSE5462) of 52 patients treated with neoadjuvant letrozole therapy [14], using SET. Five genes (in bold in Tables 3 and 4) correctly classify 26 out of 37 responders and 11 out of 15 non-responders (Fisher exact test $P$ value $=0.0056$ ).

\section{qRT-PCR results}

Four of those genes (CXCL3, GSS, FBXL18 and DUSP1) were also tested by means of RT-qPCR on a cohort of 10 FFPE breast pre-treatment biopsies from the anastrozole study, that were not used for the gene expression signature derivation. Their expression changes were in accordance with microarray analysis results, although the differences between the two groups (responders and non-responders) were not statistically significant (Supplementary Figure 2).

Changes with treatment in responder and non-responder sub-groups

A separate analysis for responders and non-responders was carried out in order to investigate whether therapy affected the expression in a different manner. The separate analysis showed that a larger number of probes were affected by the treatment in non-responders compared to responders (303 in responders and 587 in non responders). No probe was found oppositely modulated in the two groups. Most of the probes found differentially expressed in one group were not affected by the treatment in the other (only 49 probes were common between two analyses).

GO analysis of the genes differentially expressed in responders, pointed out an enrichment in GO processes, pathway maps and network processes linked to downregulation of cell cycle, stimulation of the immune 
Table 3 Genes which are upregulated in non-responders compared to responders in pretreatment samples and also are correlated with response data

\begin{tabular}{lll}
\hline & Gene symbol & Up-regulated gene description \\
\hline 1 & AGMAT & Agmatine ureohydrolase (agmatinase) \\
2 & ATXN7L2 & Ataxin 7-like 2 \\
3 & BPA-1 & Brain peptide A1 \\
4 & C17orf91 & Chromosome 17 open reading frame 91 \\
5 & C2 & Complement component 2 \\
6 & CASP4 & Caspasi 4 \\
7 & COL27A1 & Collagen, type XXVII, alpha 1 \\
$\mathbf{8}$ & CXCL3 & Chemokine (C-X-C motif) ligand 3 \\
9 & FOXS1 & Forkhead box S1 \\
10 & FUNDC2 & FUN14 domain containing 2 \\
11 & GNRHR & Gonadotropin-releasing hormone receptor \\
12 & H6PD & Hexose-6-phosphate dehydrogenase (glucose 1-dehydrogenase) \\
13 & HMX2 & H6 family homeobox 2 \\
14 & INO80D & INO80 complex subunit D \\
$\mathbf{1 5}$ & KIAA0913 & \\
16 & LOC157627 & \\
17 & MTA2 & Metastasis associated 1 family, member 2 \\
18 & NUMBL & Numb homolog (Drosophila)-like \\
19 & OR6Y1 & Olfactory receptor, family 6, subfamily Y, member \\
$\mathbf{2 0}$ & PAX4 & Paired box 4 \\
21 & RELT & Tumor necrosis factor receptor \\
22 & SFRS15 & Splicing factor, arginine/serine-rich 15 \\
23 & SPESP1 & Sperm equatorial segment protein 1 \\
24 & SPN & Sialophorin (CD43) \\
25 & TANC2 & Tetratricopeptide repeat, ankyrin repeat and coiled-coil containing 2 finger protein 157 \\
26 & ZNF157 & \\
\hline & &
\end{tabular}

unique opportunity to study molecular/genetic changes induced by therapy in breast cancer patients. However, relative few studies have been published so far on pharmacogenomic predictors of response [12-14, 18, 26, 27].

In the present study, we analyzed the molecular changes induced by anastrozole in post-menopausal breast cancer patients and looked for a signature predictive of response. For this purpose, whole genome expression profiles of biopsies collected before treatment were analyzed and associated with clinical-pathological response to treatment.

Gene ontology analysis of differentially expressed genes between pre and post-treatment samples showed that the list of down-regulated genes mainly contains features related to cell cycle progression, growth, DNA damage stimulus and DNA integrity checkpoint. Proliferation promoting genes are also differentially expressed between pre and post-treatment samples. Gene network analysis also showed that many genes transcriptionally activated by ESR1 (estrogen receptor 1) were among the down-regulated list. Similar results for down-regulated genes were also obtained by Miller et al. [18] and Mackay et al. [27].

By contrast, among up-regulated genes we found the overrepresentation of processes linked to immune system, 
Table 4 Genes which are down-regulated in nonresponders compared to responders in pre-treatment samples and also are correlated with response data

\begin{tabular}{|c|c|c|}
\hline & Gene symbol & Down-regulated gene description \\
\hline 1 & ADAMTS4 & ADAM metallopeptidase with thrombospondin type 1 motif, 4 \\
\hline 2 & AGRN & Agrin \\
\hline 3 & ARHGAP29 & Rho GTPase activating protein 29 \\
\hline 4 & CDX1 & Caudal type homeobox 1 \\
\hline 5 & COMMD10 & COMM domain containing 10 \\
\hline 6 & CPSF3L & Cleavage and polyadenylation specific factor 3-like \\
\hline 7 & DNAJB9 & DnaJ (Hsp40) homolog, subfamily B, member 9 \\
\hline 8 & DUSP1 & Dual specificity phosphatase 1 \\
\hline 9 & FBXL18 & F-box and leucine-rich repeat protein 18 \\
\hline 10 & GABRG2 & Gamma-aminobutyric acid (GABA) A receptor, gamma 2 \\
\hline 11 & GSS & Glutathione synthetase \\
\hline 12 & $\mathrm{H} 3 \mathrm{~F} 3 \mathrm{~B}$ & $\mathrm{H} 3$ histone, family 3B $(\mathrm{H} 3.3 \mathrm{~B})$ \\
\hline 13 & KTN1 & Kinectin 1 (kinesin receptor) \\
\hline 14 & LOC257396 & \\
\hline 15 & LOC285831 & \\
\hline 16 & LOC440093 & \\
\hline 17 & NAPB & $N$-Ethylmaleimide-sensitive factor attachment protein, beta \\
\hline 18 & NAPG & $\mathrm{N}$-Ethylmaleimide-sensitive factor attachment protein, gamma \\
\hline 19 & NARS2 & Asparaginyl-tRNA synthetase 2, mitochondrial (putative) \\
\hline 20 & NSUN4 & NOL1/NOP2/Sun domain family, member 4 \\
\hline 21 & OXCT1 & 3-Oxoacid CoA transferase 1 \\
\hline 22 & PCK2 & Phosphoenolpyruvate carboxykinase 2 (mitochondrial) \\
\hline 23 & PEX19 & Peroxisomal biogenesis factor 19 \\
\hline 24 & PPP1CC & Protein phosphatase 1, catalytic subunit, gamma isoform \\
\hline 25 & SAE1 & SUMO1 activating enzyme subunit 1 \\
\hline 26 & SDCBP & Syndecan binding protein (syntenin) \\
\hline 27 & TUBB2C & Tubulin, beta $2 \mathrm{C}$ \\
\hline 28 & USP13 & Ubiquitin specific peptidase 13 (isopeptidase T-3) \\
\hline
\end{tabular}

histamine metabolism and inflammation, cell adhesion and cytoskeleton, whereas previous reports only pointed out organ development and extracellular matrix turnover. In support of our findings, Wang and coworkers [28] showed that, in a rat model, anastrozole induced increased levels of proinflammatory cytokines and suppressed the differentiation of naive $\mathrm{T}$ cells to Treg cells: T-regs inhibit the antitumor functions of tumor-specific $\mathrm{T}$ cells by direct cellto-cell contact.

In general, the comparison between our results on the total cohort and Mackay's gave an overlap of 47 down- and 84 up-regulated common genes, even if Mackay's observations were after 14 days of treatment.

GO analysis of the genes differentially expressed in responders only, revealed enrichment of processes linked to down-regulation of cell cycle and to stimulation of the immune response. On the other hand, non-responders showed an increase in mechanisms of induction of T-cell energy by MHC antigen presentation without IL-2 production: these processes could reduce an immune response versus the tumor cell induced by anastrozole treatment [28].
Furthermore, non-responder tumors showed an up-regulation of network processes related to androgen receptor nuclear signaling: this suggests that tumor resistant cells could divert androgens to another cell proliferation signal to escape from aromatase cell cycle inhibition [29]. Sikora et al. [30] hypothesized that androgens and their metabolites, independently of aromatase activity, may contribute to breast cancer growth. Moreover, non-responders show a down-regulation of negative regulation of synaptic transmission, a mechanism that could be connected to cancer cell proliferation [31] and an up-regulation of Rab-regulated recycling pathways of vesicle: some elements of Rab protein family contribute to breast cancer progression [32, 33].

Our results are the first to demonstrate specific pathways involved into response to $\mathrm{AI}$, with a specific distinction between tumors that respond and those that show intrinsic resistance.

To find a signature predictive of response, probes having maximum correlation with response variable or differentially expressed between responders and non-responders were selected in pre-treatment samples. The differentially 

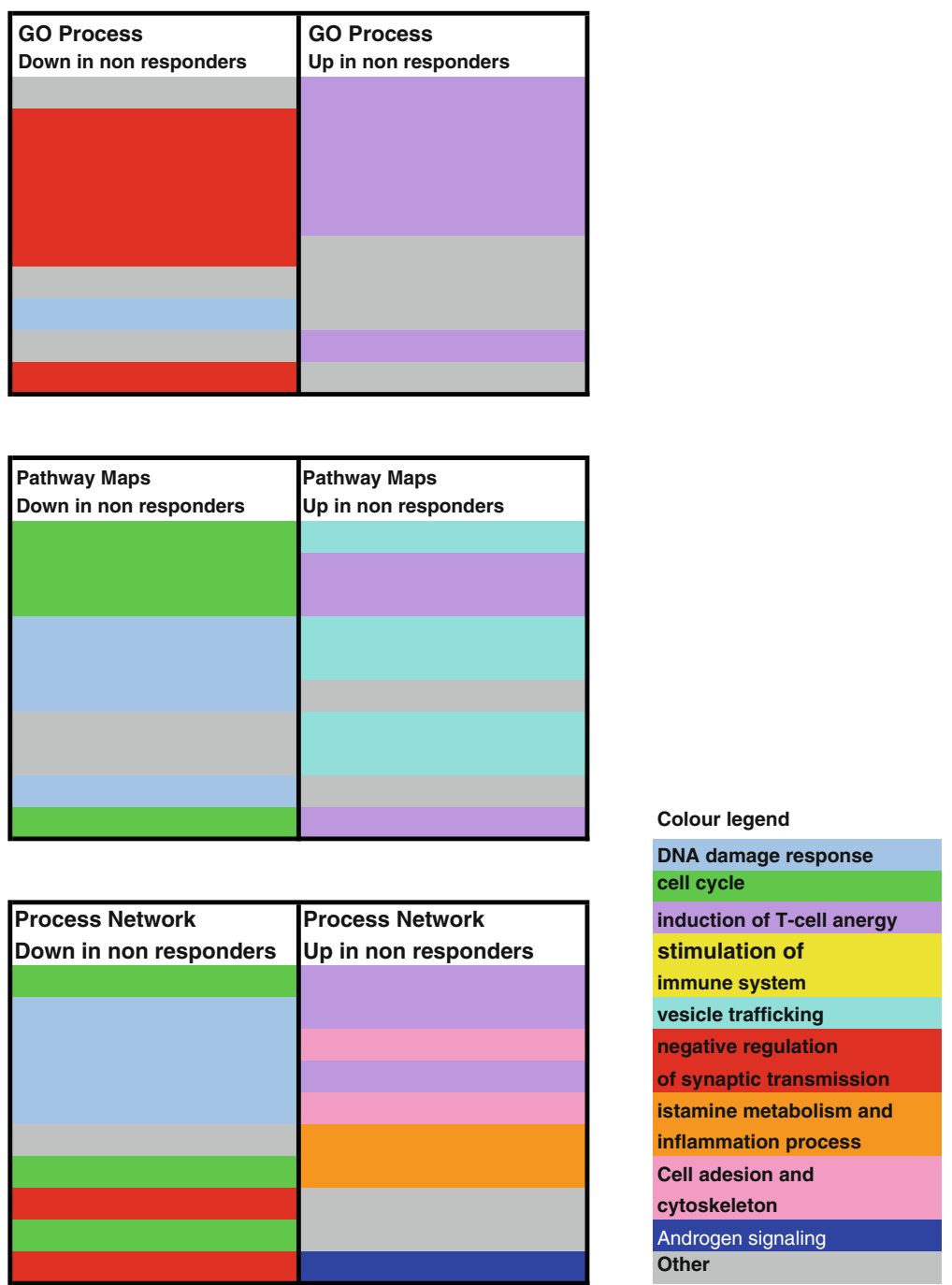
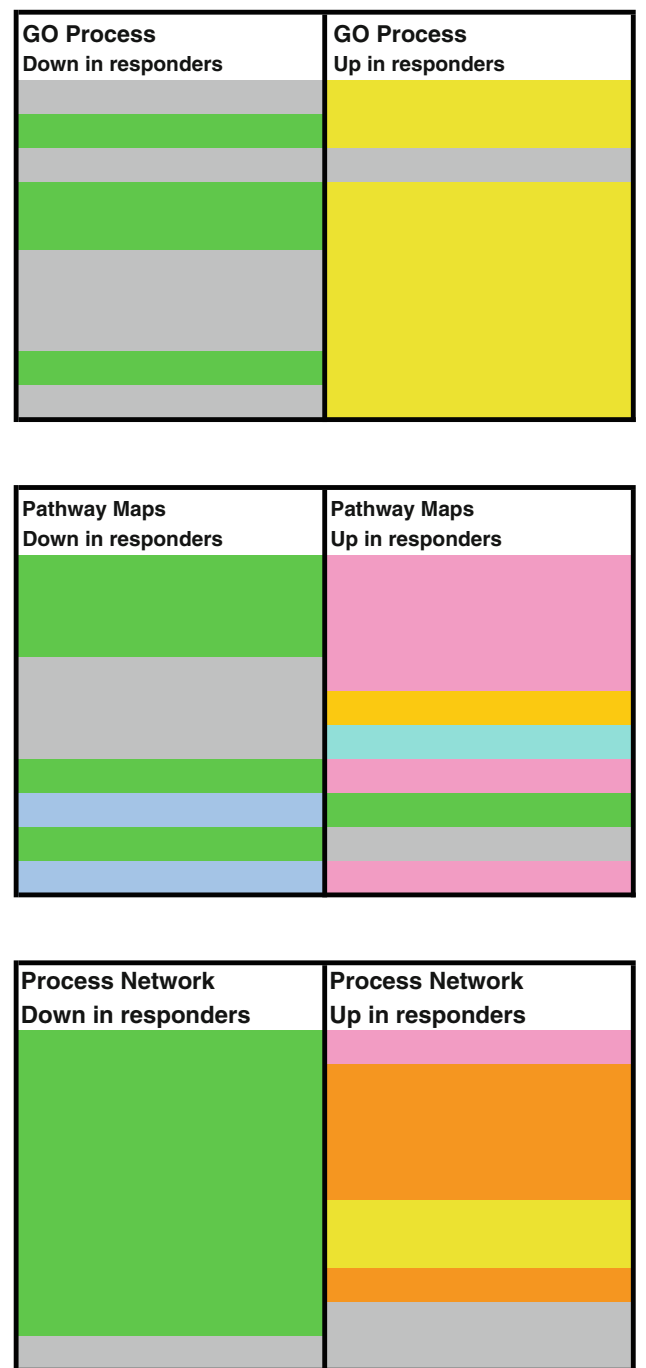

Fig. 5 Go analysis of genes differentially expressed in non-responders, and in responders. Each rectangular represents a single category and is ordered according to ascending $P$-value (as in Supplementary

expressed list performed better in separating responders from non-responders. To combine the predictive power of the two lists, probes were arranged according to the product of their ranks and the top 77 were selected as final signature (100\% accuracy). In order to test such predictor on an independent cohort, we downloaded the dataset used by Miller and coworkers [14], who derived a signature predictive of response to neoadjuvant treatment with letrozole. Our signature and the 69 pre-treatment variables in Miller's one have no common genes. However, after mapping the 77 unique Agilent probes from our analysis to 66 unique Affymetrix probes on Miller's dataset, we were able to identify six probes predictive of response to letrozole $(\mathrm{p}=0.0056)$, by using SET. The six probes correspond to five unique genes, which are CXCL3, GSS, PAX4, KIAA0913, FBXL18. Among these, CXCL3 is up-regulated in non-responders and was previously found
Table 10). Similar pathways or processes have the same color code. The right panel refers to responders while the left one to nonresponders

associated with a shorter relapse-free survival of ERalphapositive breast cancer patients treated with tamoxifen [34].

Only 17 patients satisfying the eligibility criteria discussed above completed the therapy and provided good quality RNA for microarray analysis. Due to the limited number of patients in our cohort, we used them as trainingset to derive a signature predictive of response and then used a bigger and totally independent cohort of patients with comparable characteristics as test-set. This was the only larger neoadjuvant series available to best of our knowledge to use as validation set. Although our signature derives from a small patient cohort, it succeeds in predicting response to another AI, very similar to anastrozole, in such a larger independent series.

Four of those genes were also tested on an independent cohort of 10 FFPE biopsies. Dividing patients into two groups, responders and non-responders, we obtained the 
same modulation trend as in the previous microarray analysis.

To our knowledge, a limited number of papers have been published on this subject, and none has results comparable to ours. Kristensen et al. [12] used anastrozole too, but analyzed few and heterogeneous (ER+ and ER-) patients, defining only a group of differentially expressed genes between partial response and progressive disease. Harvell et al. [13] used exemestane, alone or in combination with tamoxifen, on $7 \mathrm{ER}+$ breast cancers and individuated 50 genes associated with response; however, treatment of the 7 patients was not homogeneous: all responders were treated with exemestane only, but among non responders, three were treated with the two drugs in combination and one received exemestane only. Miller and colleagues [14] identified a combination of pre-treatment (biopsy before therapy) and changed-by-treatment (biopsy after 14 days therapy) genes able to predict response on a cohort of 52 samples treated by neoadjuvant letrozole therapy. However, it has to be emphasized that none of the former studies tested their signatures on independent datasets.

If our results will be further validated on a bigger fixed cohort, AI neoadjuvant treatment could be focused on these "likely responder" patients, pointed out by gene expression evaluation of few genes on RNA extracted from fixed biopsy. On the other hand, patients showing a "non-responsive" signature will be operated immediately, reducing risks and costs of an ineffective treatment. Molecular evaluation on tru-cut biopsy only has the advantage of avoiding treatment on patients predicted to be non-responders, without waiting for 14 days and re-biopsing the tumor, as required to translate previous results to a clinical setting.

Acknowledgements We thank Dr Annalisa Lanza, from Fondazione Maugeri, for sample storing and delivery and Prof Paolo Provero (Molecular Biotechnology Centre, University of Torino) for his supervision on data analysis. The study was partially supported by an educational grant from Astra Zeneca, who had no role in study design, data collection or interpretation, or manuscript submission, for which the authors had full responsibility.

\section{References}

1. Hortobagyi GN (1998) Treatment of breast cancer. N Engl J Med 339:974-984

2. Early Breast Cancer Trialists' Collaborative Group (1998) Tamoxifen for early breast cancer: an overview of the randomised trials. Lancet 351:1451-1467

3. Mauriac L, Smith I (2003) Aromatase inhibitors in early breast cancer treatment. Semin Oncol 4(Suppl 14):46-57

4. Mouridsen H, Gershanovich M, Sun Y, Pérez-Carrión R, Boni C et al (2001) Superior efficacy of letrozole versus tamoxifen as first-line therapy for postmenopausal women with advanced breast cancer: results of a phase III study of the International Letrozole Breast Cancer Group. J Clin Oncol 19:2596-2606
5. Goss PE, Ingle JN, Martino S, Robert NJ, Muss HB et al (2003) A randomized trial of letrozole in postmenopausal women after five years of tamoxifen therapy for early-stage breast cancer. N Eng J Med 349:1793-1802

6. Coombes RC, Hall E, Gibson LJ, Paridaens R, Jassem J et al (2004) A randomized trial of exemestane after two to three years of tamoxifen therapy in postmenopausal women with primary breast cancer. N Engl J Med 350:1081-1092

7. Harada N (1997) Aberrant expression of aromatase in breast cancer tissues. J Steroid Biochem Mol Biol 61:175-184

8. Sun XZ, Zhou D, Chen S (1997) Autocrine and paracrine actions of breast tumor aromatase. A three-dimensional cell culture study involving aromatase transfected MCF-7 and T-47D cells. J Steroid Biochem Mol Biol 63:29-36

9. Miller WR, Dixon JM, Macfarlane L, Cameron D, Anderson TJ (2003) Pathological features of breast cancer response following neoadjuvant treatment with either letrozole or tamoxifen. Eur J Cancer 39:462-468

10. Anderson TJ, Dixon JM, Stuart M, Sahmoud T, Miller WR (2002) Effect of neoadjuvant treatment with Anastrozole on tumour histology in postmenopausal women with large operable breast cancer. Br J Cancer 87:334-338

11. Milla Santos A, Milla 1, Calvo N, Portella J, Rallo L et al (2004) Anastrozole as neoadjuvant therapy for patients with hormonedependent, locally-advanced breast cancer. Anticancer Res 24:1315-1318

12. Kristensen VN, Sørlie T, Geisler J, Yoshimura N, Linegjaerde OC et al (2005) Effects of Anastrozole on the intratumoral gene expression in locally advanced breast cancer. J Steroid Biochem Mol Biol 95:105-111

13. Harvell DM, Richer JK, Singh M, Spoelstra N, Finlayson C et al (2008) Estrogen regulated gene expression in response to neoadjuvant endocrine therapy of breast cancer: tamoxifene agonist effects dominate in the presence of an aromatase inhibitor. Breast Cancer Res Treat 112:489-501

14. Miller WR, Larionov A, Renshaw L, Anderson TJ, Walker JR et al (2009) Gene expression profiles differentiating between breast cancers clinically responsive or resistant to letrozole. J Clin Oncol 27:1382-1387

15. Therasse P, Arbuck SG, Eisenhauer EA, Wanders J, Kaplan RS et al (2000) New guidelines to evaluate the response to treatment in solid tumors. European Organization for Research and Treatment of Cancer, National Cancer Institute of the United States, National Cancer Institute of Canada. J Natl Cancer Inst 92: 205-216

16. Deaglio S, Vaisitti T, Aydin S, Bergui L, D’Arena G et al (2007) CD38 and ZAP-70 are functionally linked and mark CLL cells with high migratory potential. Blood 110:4012-4021

17. Smyth GK (2004) Linear models and empirical bayes methods for assessing differential expression in microarray experiments. Stat Appl Genet Mol Biol 3: Article 3

18. Miller WR, Larionov A, Renshaw L, Anderson TJ, White S et al (2007) Aromatase inhibitors-gene discovery. J Steroid Biochem Mol Biol 106:130-142

19. Scatolini M, Mello Grand M, Grosso E, Venesio T, Pisacane A et al (2010) Altered molecular pathways in melanocytic lesions. Int J Cancer 126:1869-1881

20. Ochsner SA, Steffen DL, Hilsenbeck SG, Chen ES, Watkins C, McKenna NJ (2009) GEMS (Gene Expression MetaSignatures), a Web resource for querying meta-analysis of expression microarray datasets: 17beta-estradiol in MCF-7 cells. Cancer Res 69:23-26

21. Itoh T, Karlsberg K, Kijima I, Yuan YC, Smith D et al (2005) Letrozole-, Anastrozole-, and tamoxifen-responsive genes in MCF-7aro cells: a microarray approach. Mol Cancer Res 3: 203-218 
22. Hayashi S, Yamaguchi Y (2008) Estrogen signaling in cancer microenvironment and prediction of response to hormonal therapy. J Steroid Biochem Mol Biol 109:201-206

23. Williams C, Edvardsson K, Lewandowski SA, Ström A, Gustafsson JA (2008) A genome-wide study of the repressive effects of estrogen receptor beta on estrogen receptor alpha signaling in breast cancer cells. Oncogene 27:1019-1032

24. Sismondi P, Biglia N, Ponzone R, Fuso L, Scafoglio C et al (2007) Influence of estrogens and antiestrogens on the expression of selected hormone-responsive genes. Maturitas 57:50-55

25. Wilson CL, Sims AH, Howell A, Miller CJ, Clarke RB (2006) Effects of oestrogen on gene expression in epithelium and stroma of normal human breast tissue. Endocr Relat Cancer 13:617-628

26. Cappelletti V, Gariboldi M, De Cecco L, Toffanin S, Reid JF et al (2008) Patterns and changes in gene expression following neoadjuvant anti-estrogen treatment in estrogen receptor-positive breast cancer. Endocr Relat Cancer 15:439-449

27. Mackay A, Urruticoechea A, Dixon JM, Dexter T, Fenwick K et al (2007) Molecular response to aromatase inhibitor treatment in primary breast cancer. Breast Cancer Res 9:R37

28. Wang J, Zhang Q, Jin S, Feng M, Kang X et al (2009) Immoderate inhibition of estrogen by anastrozole enhances the severity of experimental polyarthritis. Exp Gerontol 44:398-405
29. Lin HY, Sun M, Lin C, Tang HY, London D et al (2009) Androgen-induced human breast cancer cell proliferation is mediated by discrete mechanisms in estrogen receptor-alphapositive and -negative breast cancer cells. J Steroid Biochem Mol Biol 113:182-188

30. Sikora MJ, Cordero KE, Larios JM, Johnson MD, Lippman ME et al (2009) The androgen metabolite 5alpha-androstane-3beta, 17beta-diol (3betaAdiol) induces breast cancer growth via estrogen receptor: implications for aromatase inhibitor resistance. Breast Cancer Res Treat 115:289-296

31. Español AJ, de la Torre E, Fiszman GL, Sales ME (2007) Role of non-neuronal cholinergic system in breast cancer progression. Life Sci 80:2281-2285

32. Jones MC, Caswell PT, Norman JC (2006) Endocytic recycling pathways: emerging regulators of cell migration. Curr Opin Cell Biol 18:549-557

33. Stein MP, Dong J, Wandinger-Ness A (2003) Rab proteins and endocytic trafficking: potential targets for therapeutic intervention. Adv Drug Deliv Rev 55:1421-1437

34. Bièche I, Chavey C, Andrieu C, Busson M, Vacher S et al (2007) $\mathrm{CXC}$ chemokines located in the $4 \mathrm{q} 21$ region are up-regulated in breast cancer. Endocr Relat Cancer 14:1039-1052 